\title{
Sleep disorders and cerebrovascular disease: the long and winding road
}

\author{
Jean Louis Pepin ${ }^{1}$ and Miguel Angel Martinez-Garcia ${ }^{2}$ \\ Affiliations: 'Université Grenoble Alpes, Inserm, CHU Grenoble Alpes, Grenoble, France. ${ }^{2}$ Pneumology Dept, \\ Polytechnic and University La Fe Hospital, Valencia, Spain.
}

Correspondence: Jean-Louis Pepin, Laboratoire EFCR, CHU Grenoble Alpes, CS10217, 38043 Grenoble Cedex 9. France. E-mail: JPepinđachu-grenoble.fr

\section{@ERSpublications}

The association between stroke and OSA cerebrovascular disease http://bit.ly/33ZnCKy

Cite this article as: Pepin JL, Martinez-Garcia MA. Sleep disorders and cerebrovascular disease: the long and winding road. Eur Respir J 2020; 55: 1901977 [https://doi.org/10.1183/13993003.01977-2019].

\begin{abstract}
A human being spends (or at least should spend) around a third of their life asleep. During normal sleep, physiological changes occur in the neurological, metabolic and cardiovascular systems, and these serve to maintain good health during waking hours [1]. Some specific diseases arise only during sleep, while others are most frequently identified, and most susceptible to aggravation, at night. The lifestyle imposed by our contemporary societies is not only associated with obesity and a diabetes epidemic but also with a massively increased prevalence of chronic sleep deprivation and sleep disorders, with an estimated one billion people suffering from sleep apnoea worldwide [2]. Insomnia and sleep disordered breathing are now two of the most common chronic diseases capable of aggravating the natural history of cardio-metabolic diseases.

Reduction in sleep duration, sleep fragmentation and intermittent hypoxia during sleep are the main triggers for intermediary mechanisms such as sympathetic activation, oxidative stress and low-grade inflammation that can increase the incidence and severity of cardiovascular and metabolic diseases [3]. Previous studies have reported that stroke and transient ischaemic attacks occur more frequently at night or soon after waking up (wake-up strokes), particularly when associated sleep disorders are also present [4]. Beyond these potential pathophysiological mechanisms underlying the association between stroke and sleep disorders, clinical studies have failed to provide any conclusive proof of a causal relationship between sleep disorders and cerebrovascular diseases, due to methodological issues and the various confounders and heterogeneity present in the populations studied to date [5]. Large, well-designed, randomised interventional studies are therefore still needed.
\end{abstract}

The current issue of the European Respiratory Journal presents a consensus statement that addresses this important clinical question in depth [6]. Using strict methodology, the statement gathers together all the scientific information available on the relationship between sleep disorders and stroke, two diseases of undeniable interest to general public health owing to their prevalence and associated disability and health-related costs [7]. This statement has the further advantage of covering not only sleep apnoea but also other frequent sleep disorders, including insomnia and restless leg syndrome/periodic limb movements. The conclusions will be widely disseminated as the document represents a multidisciplinary consensus reached by 15 experts from four different scientific societies and organisations with links to the fields of sleep disorders and cerebrovascular disease (European Respiratory Society, European Academy of Neurology, European Sleep Research Society and European Stroke Organization). 
The authors' methodology was appropriate to the collection and classification of the most relevant known information, with 13 questions identified as being of crucial clinical importance. There was no evidence-based demonstration of any significant link between insomnia, the intake of psychoactive drugs (particularly benzodiazepines and their derivatives) or restless leg syndrome and incident cerebrovascular disease. This conclusion is accompanied by a call to research groups to undertake further studies in this field.

There seems to be more information available (albeit less than could be desired) on the relationship between sleep disordered breathing (particularly its predominant manifestations: obstructive sleep apnoea (OSA)) and cerebrovascular disease. Most studies have found an increase in the prevalence and severity of OSA in both patients with an acute stroke and those in a stable phase [8], and this association could have an impact on both functional outcomes after stroke and the recurrence of any cardiovascular event [9]. The primary treatment of OSA (especially with continuous positive airway pressure (CPAP)) seems to provide some protection against stroke recurrence in adherent patients [10].

The significant association between both prevalent and incident stroke and OSA in clinical studies suggests that the detection and treatment of the former requires more attention in the special groups of patients with risk factors for stroke, and even more so in those with established stroke. Some areas of particular importance would be: 1) the potential protective role of CPAP treatment in patients with transient ischaemic attacks, in order to avoid future established strokes; 2) OSA management during the acute phase of stroke, as the cerebral hypoxic penumbra can still be recovered during this period and brain remodelling can occur (this is linked with oxidative stress and inflammation, which can be aggravated by untreated sleep apnoea [11]); 3) changes in central and obstructive respiratory events from the acute to the stable phase of stroke, to determine the best schedule for conducting a diagnostic sleep study and initiating positive airway pressure therapy; 4) analysis of the impact of OSA and CPAP treatment on well-delineated series of haemorrhagic strokes, since the risk factors and OSA impact differ from those of ischaemic strokes; and, finally; 5) assessment of the impact of the combination of OSA and other sleep disorders is of particular interest. The insomnia-sleep apnoea phenotype is highly prevalent and is associated with low CPAP compliance and poor outcomes. This is also true for periodic limb movements and sleep apnoea. Recent data have demonstrated that such associations are linked with a higher risk of subsequent cardiovascular events, including stroke [12].

One of the main obstacles to the implementation of studies in this field is the large number of confounders involved (and the complexity of their interrelationships), as well as the great heterogeneity found in the presentation of both sleep disorders and strokes. These factors would make it necessary to study a number of specific subgroups (phenotypes) [5]. Sleep disorders (whether associated with breathing disorders or not) tend to appear more frequently in the elderly, and this is also the case with stroke. In this population, various comorbidities, such as the ingestion of several drugs (for both sleep disorders and cardiovascular disease prevention) and even the coexistence of several cardiovascular risk factors, can make it difficult to establish any clear relationship between the different diseases [12]. In fact, some authors have pointed out that this could be one of the reasons why the significance of associations between these diseases is usually more commonly reported in younger patients, where confounders are much less prominent [5].

However, although there is undoubtedly a long and winding road ahead of us, it is clear that, of all the studies undertaken to date on the relationship between OSA and cardiovascular disease, the ones with the most positive and promising results have focused on the effect of CPAP on cerebrovascular disease. We should therefore prioritise this aspect. This should encourage the community to implement a well-designed, multinational clinical study on the impact of CPAP on incident stroke (adherence should be checked and improved during a run-in period). The current task force involving four societies and a panel of experts would constitute a solid foundation for such an initiative.

Conflict of interest: None declared.

\section{References}

Collop NA, Salas RE, Delayo M, et al. Normal sleep and circadian processes. Crit Care Clin 2008; 24: 449-460.

2 Benjafield AV, Ayas NT, Eastwood PR, et al. Estimation of the global prevalence and burden of obstructive sleep apnoea: a literature-based analysis. Lancet Resp Med 2019; 7: 687-698.

3 Javaheri S, Barbe F, Campos-Rodriguez F, et al. Sleep apnea: types, mechanisms, and clinical cardiovascular consequences. J Am Coll Cardiol 2017; 69: 841-858.

4 Xiao Z, Xie M, You Y, et al. Wake-up stroke and sleep-disordered breathing: a meta-analysis of current studies. J Neurol 2018; 265: 1288-1294.

5 Javaheri S, Martinez-Garcia MA, Campos-Rodriguez F. CPAP treatment and cardiovascular prevention: we need to change the design and implementation of our trials. Chest 2019; 156: 431-437. 
6 Bassetti CLA, Randerath W, Vignatelli L, et al. EAN/ERS/ESO/ESRS statement on the impact of sleep disorders on risk and outcome of stroke. Eur Respir J 2020; 55: 1901104.

7 Nguyen G, Cercy K, Johnson CO, et al. Global, regional, and country-specific lifetime risk of stroke 1990 and 2016. N Engl J Med 2018; 379: 2429-2437.

8 Johnson KG, Johnson DC. Frequency of sleep apnea and stroke and TIA patients: a meta-analysis. J Clin Sleep Med 2010; 6: 131-137.

9 Li M, Hou WS, Zhang XW, et al. Obstructive sleep apnea and risk of stroke: a meta-analysis of prospective studies. Int J Cardiol 2014; 172: 466-469.

10 Brill AK, Horvath T, Seiler A, et al. CPAP as treatment of sleep apnea after stroke: A meta-analysis of randomized trials. Neurology 2018; 90: e1222-e1230.

11 Moskowitz MA, Lo EH, Iadecola C. The science of stroke: mechanism in search of treatment. Neuron 2010; 67: 181-198.

12 Zinchuk AV, Jeon S, Koo BB, et al. Polysomnographic phenotypes and their cardiovascular implications in obstructive sleep apnoea. Thorax 2018; 73: 472-480. 